\title{
Preoperative Ultra-Short-Course Chemotherapy Combined with Surgery for the Treatment of Chest Wall Tuberculosis
}

This article was published in the following Dove Press journal: Infection and Drug Resistance

\section{Weidong Zhang \\ Jingfang Chen \\ Xiaoming Wu \\ Luyu Chen \\ Jinxing Wei \\ Mingqiang Xue \\ Yuanyuan Liu \\ Qingzheng Liang}

Department of Thoracic Surgery, Henan Provincial Chest Hospital, Zhengzhou 450003, Henan Province, People's

Republic of China
Correspondence: Weidong Zhang;

Qingzheng Liang

Email zhangweidong037I@I26.com;

707382852@qq.com
Objective: To investigate the safety and efficacy of preoperative ultra-short-course chemotherapy, combined with surgical treatment for chest wall tuberculosis and summarize our experience in this regard, to provide a reference for national and international clinicians.

Methods: A retrospective analysis was conducted of the clinical data, preoperative antituberculosis duration, and postoperative recurrence rate in 263 patients with chest wall tuberculosis spanning 5 years.

Results: Overall, 263 patients were treated with anti-tuberculosis drugs for about $\overline{17.06} \pm$ 12.49 days during the preoperative period. Simple chest wall tuberculosis was treated for $\overline{12.82} \pm 5.87$ days and composite chest wall tuberculosis for $\overline{21.04} \pm 5.11$ days. The postoperative recurrence rate of chest wall tuberculosis was $3.80 \%$, which was close to or lower than the recurrence rate of routine preoperative anti-tuberculous therapy in patients subjected to ultra-short-range anti-tuberculosis treatment before surgery.

Conclusion: Preoperative ultra-short-course chemotherapy combined with surgical treatment of chest wall tuberculosis did not increase the recurrence rate of chest wall tuberculosis; moreover, it could effectively shorten hospitalization time and improve patient compliance. Full-line anti-tuberculosis treatment and complete resolution of tuberculosis infections are crucial to curing chest wall tuberculosis.

Keywords: chest wall tuberculosis, surgical treatment, ultra-short-course chemotherapy

\section{Introduction}

Chest wall tuberculosis (TB) is a relatively rare form of extrapulmonary TB, accounting for about $1-2 \%$ of all TB cases. ${ }^{1-3}$ Because its incidence is unknown, the diagnosis difficult, and the treatment methods diverse, there is poor consensus among medical workers at different levels, resulting in most patients having to face a longer course of disease, higher recurrence rate, and torturous treatment process. Several studies, both local and international, ${ }^{4-9}$ have suggested surgical treatment for chest wall TB. Surgical removal of the tuberculous focus has gradually become the mainstream treatment method for chest wall TB.

However, at present, most researchers ${ }^{8,10,11}$ advocate at least a 2-3-month-long preoperative anti-TB treatment. This long-term anti-TB treatment before the operation greatly increases patient discomfort and reduces compliance. In recent years, our hospital has carried out a preoperative ultra-short-term anti-TB program combined with surgery for the treatment of chest wall TB, with a high success rate and low 
recurrence rate. A total of 263 patients with chest wall TB treated at our hospital from 2013 to 2018 were retrospectively analyzed. The clinical data, preoperative anti-TB duration, and postoperative recurrence rate were analyzed to evaluate the effects of preoperative ultra-short-term anti-TB treatment with regard to surgical safety and the curative effect.

\section{Materials and Methods}

\section{General Information}

The study was conducted from January 2013 to June 2018. We identified and included patients who underwent "chest wall tuberculous focus debridement" at our hospital. Patients with chest wall TB from intrathoracic tuberculous pleurisy or tuberculous empyema were excluded (Table 1). A total of 263 patients were included (176 male and 87 female patients; median age: 31 [24-48] years). All patients were divided into two groups, the simple chest wall TB group $(n=119)$ and the composite chest wall TB group $(n=144)$. The composite chest wall TB group was defined as patients with chest wall TB that was concomitant with other types of TB or other TB history. They included the following types: pulmonary TB $(\mathrm{n}=88)$; tuberculous pleurisy $(\mathrm{n}=36)$; vertebral TB $(\mathrm{n}=10)$; lymph node TB $(\mathrm{n}=8)$; and abdominal wall TB $(\mathrm{n}=2)$.

Among the study participants, TB of the anterior, lateral, and posterior chest wall was identified in 164, 68, and 31 patients, respectively. Overall, there were 59 cases of sinus formation caused by TB lesions piercing the chest wall (Figure 1A) and 49 cases of dumbbell-shaped abscesses due to TB invasion of the intercostal muscles (Figure 1B). The surgical incisions of another 48 patients, who underwent surgery at another hospital, did not heal and secreted pus (Figure 1C and D). In the remaining 107 patients, only the formation of local lumps was observed on the chest wall (Figure 1E).

\section{Principles of Preoperative Management}

After patients were admitted to the hospital, puncture biopsies were performed to confirm the diagnosis of TB. For patients with negative pathological results, we conducted tests for TB antibodies, tuberculin skin experiments, T-SPOT tests, and other TB-related tests to confirm the diagnosis. All patients were diagnosed according to the Chinese TB diagnostic criteria (WS 288-2017). ${ }^{12}$ Furthermore, the diagnosis of all patients was verified by examining postoperative pathological specimens. Patients with a confirmed diagnosis were carefully examined after admission to avoid the omission of other lesions, and a general preoperative examination was performed to determine the existence of any surgical contraindications.

Chest computed tomography (CT) was performed to determine the location, scope, and depth of the lesion; ie, whether a dumbbell-shaped lesion was formed, whether the abscess penetrated the chest, whether the ribs and costal cartilage were destroyed, and whether the lungs and pleura were involved in the infection.

After a thorough evaluation of all patients' cardiopulmonary function, we confirmed that all were able to tolerate surgical treatment. Among them, 13 patients showed varying degrees of liver injury, owing to externally prescribed anti-TB drugs. These patients underwent surgery for TB only after correction of liver function and adequate liver-protective treatment. A total of 30 patients were considered to be in the active stage of TB, including 25 cases of pulmonary and pleural TB, and five cases of chest wall TB alone. The patients with active TB were treated with enhanced anti-TB therapy immediately after admission (following a similar schedule as the other patients).

All patients were treated with the following intensive anti-TB regimen immediately after admission: rifampicin (600 $\mathrm{mg}$ ) and isoniazid (300 $\mathrm{mg}$ ) as intravenous drips once a day, and oral ethambutol (750 $\mathrm{mg})$ and pyrazinamide $(1500 \mathrm{mg})$ once every night. For patients with retreated $\mathrm{TB}$, the regimen included amikacin (600 $\mathrm{mg})$, sodium p-aminosalicylate (10 g), and levofloxacin (600 mg) as intravenous drips administered once a day.

When drug-sensitivity testing for TB bacteria was determined, more than four types of sensitive anti-TB drugs were selected, according to the results of the drug-sensitivity test. Prophylactic oral liver-protection therapy was simultaneously administered with anti-TB treatment. Some patients with symptoms of nausea, vomiting, and loss of appetite were treated with gastric protection and antiemetic drugs, and pyrazinamide and sodium p-aminosalicylate were discontinued.

Patients with hepatic injury were given an intravenous infusion of a variety of liver-protection drugs. Other patients with hypertension, diabetes, and coronary heart disease were treated with appropriate medications. The preoperative anti-TB duration for all patients was $\overline{17.06}$ \pm 12.49 days (range: $7-80$ days) (Table 2). During the period of preoperative anti-TB treatment, 196 patients with chest wall TB lesions showed improvement to varying degrees, such as reduction and closure of the sinus, 
Table I General Clinical Data of the 263 Included Cases

\begin{tabular}{|c|c|c|c|c|}
\hline \multirow[t]{2}{*}{ Characteristics } & $\begin{array}{l}\text { Simple Chest Wall } \\
\text { Tuberculosis }\end{array}$ & $\begin{array}{l}\text { Composite Chest Wall } \\
\text { Tuberculosis }\end{array}$ & $\begin{array}{l}\text { All } \\
\text { Patients }\end{array}$ & \multirow[t]{2}{*}{$P$ value } \\
\hline & $(n=119)$ & $(n=144)$ & $(n=263)$ & \\
\hline Age, years [median (IQR)] & $32(25-48)$ & $29(23-47)$ & $31(24-48)$ & 0.1460 \\
\hline \multicolumn{5}{|l|}{$\operatorname{Sex}(n)$} \\
\hline Male & 83 & 93 & 176 & 0.1257 \\
\hline Female & 36 & 51 & 87 & \\
\hline \multicolumn{5}{|l|}{ Lesion location (n) } \\
\hline Anterior chest wall & 71 & 93 & 164 & 0.3724 \\
\hline Lateral chest wall & 31 & 37 & 68 & \\
\hline Posterior chest wall & 17 & 14 & 31 & \\
\hline \multicolumn{5}{|l|}{ Local feature (n) } \\
\hline Local lumps & 74 & 81 & 154 & \\
\hline Sinus tract & 25 & 34 & 59 & 0.7524 \\
\hline Dumbbell-shaped abscess & 20 & 29 & 49 & \\
\hline \multicolumn{5}{|l|}{ History of prior treatment } \\
\hline Operation history $(n)^{*}$ & 36 & 12 & 48 & \\
\hline \multicolumn{5}{|c|}{ Other sites' tuberculosis history } \\
\hline Pulmonary tuberculosis & & 88 & 88 & \\
\hline Tuberculous pleurisy & & 36 & 36 & \\
\hline Vertebral tuberculosis & & 10 & 10 & \\
\hline Lymph node tuberculosis & & 8 & 8 & \\
\hline Abdominal tuberculosis & & 2 & 2 & \\
\hline \multirow{2}{*}{\multicolumn{5}{|c|}{$\begin{array}{l}\text { Pre-operation anti-tuberculosis } \\
\text { time (days) }\end{array}$}} \\
\hline & & & & \\
\hline \multicolumn{5}{|l|}{ Distribution (n) } \\
\hline $1-7$ & 8 & 10 & 18 & 0.4911 \\
\hline $8-14$ & 72 & 60 & 132 & \\
\hline$|5-2|$ & 25 & 33 & 58 & \\
\hline $22-28$ & 14 & 13 & 27 & \\
\hline$>28 d$ & 0 & 28 & 28 & \\
\hline \multicolumn{5}{|c|}{ Postoperative hospital stay (days) } \\
\hline \multicolumn{5}{|l|}{ Distribution (n) } \\
\hline $7-14$ & 7 & 21 & 28 & 0.3393 \\
\hline$|5-2|$ & 55 & 55 & 110 & \\
\hline $22-28$ & 48 & 42 & 90 & \\
\hline$>28$ & 9 & 26 & 35 & \\
\hline Recurrence (n) & 3 & 7 & 10 & \\
\hline
\end{tabular}

Note: *The surgery for chest wall tuberculosis included simple abscess removal, incision, and drainage.

gradual reduction of empyema-incision secretion, reduction of TB lesion, and relief from pain and discomfort.

\section{Surgical Methods}

All patients underwent debridement of chest wall TB under general anesthesia. Most patients were given a laryngeal mask, combined with intravenous anesthesia.
Only some patients needed tracheal intubation combined with intravenous anesthesia, particularly if they needed to undergo a chest operation due to lesion rupture through the parietal pleura.

The surgical incision was based on preoperative chest CT positioning, and was made mostly at the midpoint of the lesion, or a slightly upward horizontal incision (for those 

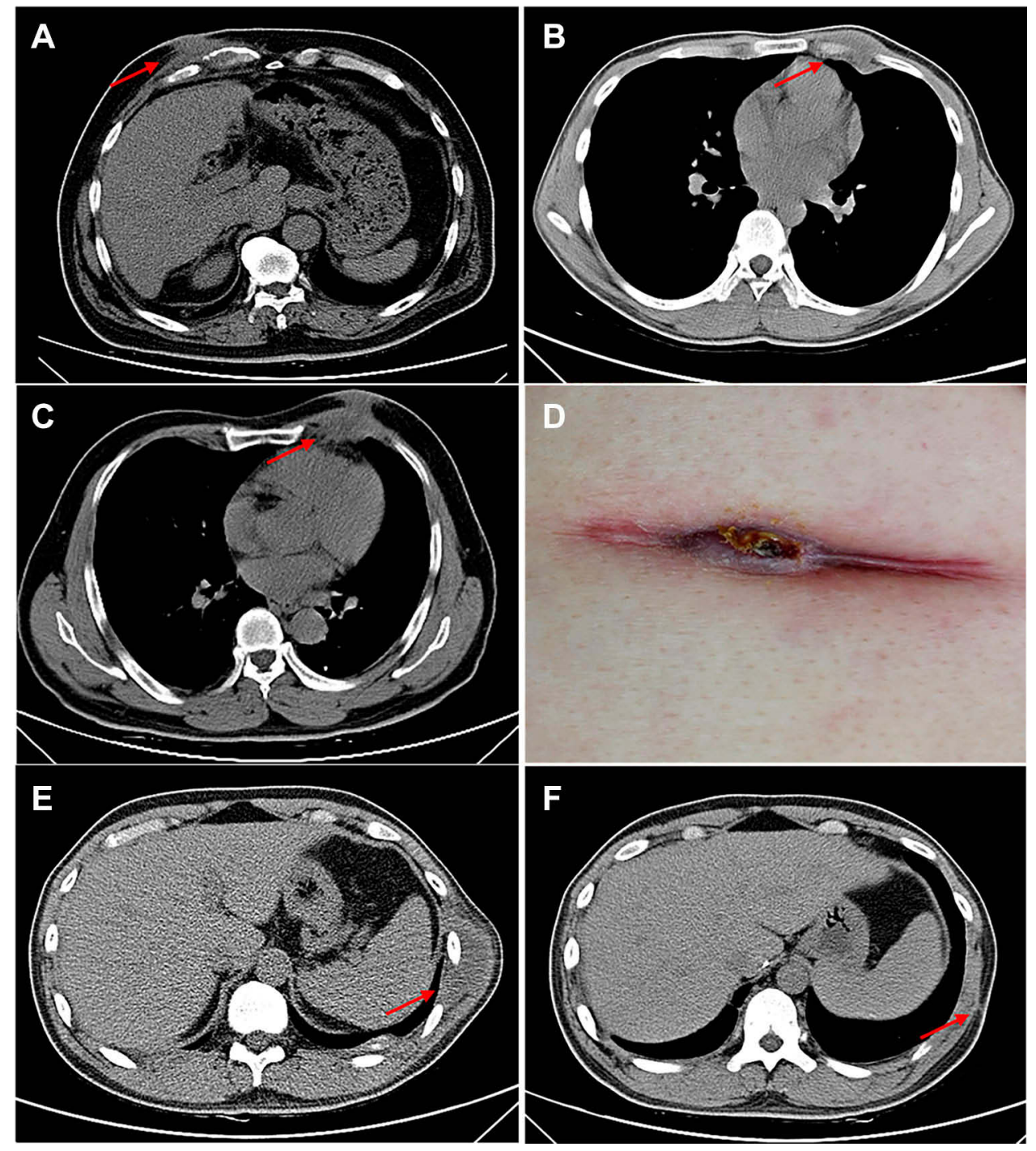

Figure I Imaging findings of chest wall tuberculosis (TB). (A) Sinus formation caused by TB lesions piercing the chest wall. (B) Chest wall TB lesion breached through the intercostal muscle to form a dumbbell-like abscess. (C) In some patients with chest wall TB, the lesion showed no signs of healing after surgical treatment (no anti-TB treatment). (D) Secretion at the incision site. (E) Chest wall TB lesion only formed in local thoracic masses. (F) After the patient depicted in panel E underwent chest wall lesion removal, his lesion showed no residue and the wound healed well.

with a smaller chest wall mass). An "S"-shaped incision was made for larger chest wall abscesses and a fusiform incision was made for those with previous surgical scars or ulceration. After opening the skin tissue of the lesion, it was fully exposed to enable systematic removal of local necrotic material and granulomatous tissue.

The sinus shape was carefully explored, and the sinus was then cut and curetted to remove the diseased tissue. For

Table 2 Anti-Tuberculosis Time Comparison

\begin{tabular}{|l|l|l|}
\hline & Preoperative Anti-Tuberculosis Time (Day) & Postoperative Hospital Stay (Day) \\
\hline Simple chest wall tuberculosis & $12.82^{\mathrm{a}} \pm 5.87$ & $21.04^{\mathrm{b}} \pm 5.11$ \\
\hline Composite chest wall tuberculosis & $20.57 \pm 15.16$ & $22.42 \pm 9.68$ \\
\hline All chest wall tuberculosis & $17.06 \pm 12.49$ & $21.91 \pm 7.89$ \\
\hline
\end{tabular}

Note: ${ }^{\mathrm{a}} \mathrm{P}<0.0001,{ }^{\mathrm{b}} \mathrm{P}=0.0868$. 
patients showing destruction of the ribs and costal cartilage, and for those with the sinus extending below the ribs and costal cartilage such that it could not have been explored, the best approach was to resect part of the ribs and costal cartilage and completely remove the intercostal intramuscular lesion and necrosed tissue. Before the end of the operation, the incisions of all patients were flushed with $3 \%$ hydrogen peroxide and warm saline in turn, and local rifampicin (600 mg) and isoniazid (300 mg).

For patients with large residual cavities, the surrounding muscle tissue had to be released to fill the residual cavity and isolate the ribs and costal cartilage stumps. If necessary, a drainage tube was inserted into the residual cavity and connected to a negative pressure device. The incision was closed after suturing the functional muscle layers such as the serratus anterior muscle, and latissimus dorsi muscle. After the operation, pressure bandages were applied to the chest to prevent residual cavity effusion, or the recurrence of infection, and promote healing. The purulent fluid removed during the operation was routinely cultured for TB, and drug-sensitivity tests for TB bacteria were performed after the operation.

\section{Postoperative Management}

All patients were examined for exudation from the incisions about 3 days after the operation. If there was no obvious exudation, wound healing was evaluated by changing the dressing every week, and the sutures were removed about 10-16 days after the operation. However, if there was obvious exudation or a poor blood supply, wound healing was monitored every 2-3 days, and suture removal was appropriately postponed. Pressure bandages were repeatedly applied to the surgical wounds until about 21 days after surgery.

After the operation, patients continued to be treated at the hospital with the same preoperative anti-TB regimen, until the wounds were healed, sutures were removed, and

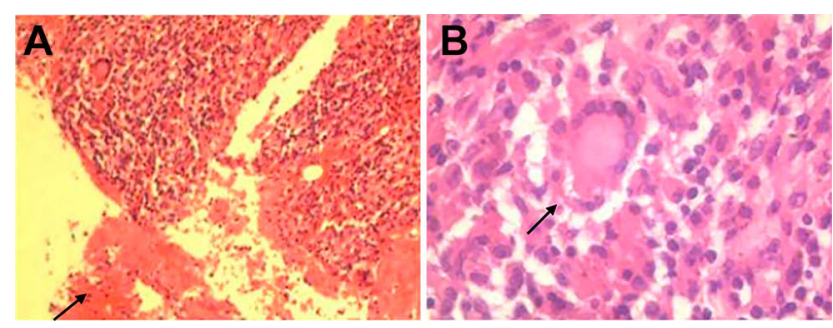

Figure 2 Histopathological sections of chest wall TB lesions, showing necrotic material (A) and granulomatous lesions with multinucleated giant cells (B). Pathology confirmed that the lesion was tuberculous granulomatous inflammation. the characteristic TB symptoms were completely resolved. At that point, they were discharged. After discharge, they continued to receive regular anti-TB treatment $(2 \mathrm{HRZE} /$ 10HRE, or according to the drug-sensitivity results). Patients were also prescribed oral liver-protection therapy. Furthermore, they underwent monthly blood tests to assess liver and kidney function and bone marrow suppression. Based on these results, an individually tailored anti-TB drug regimen was prescribed.

\section{Statistical Methods}

All statistical analyses were conducted using the SPSS21.0 software. The preoperative anti-TB duration and postoperative hospital stay between patients with simple and composite chest wall TB were compared using the pairwise Fisher's test. $\mathrm{P}<0.05$ was considered to indicate statistical significance. Quantitative data of both groups were presented as the mean and standard deviation.

\section{Results}

All 263 patients showed favorable wound healing (Figure 1F). The postoperative pathological report of the patients indicated tuberculous granulomatous inflammation (Figure 2), and a confirmed diagnosis of chest wall TB was evident. All patients continued to receive regular anti-TB treatment after discharge. The mean time of strengthening anti-TB therapy before the operation was $\overline{17.06} \pm 12.49$ days (range: 7-80 days), including $\overline{12.82} \pm 5.87$ days (range: 7-28 days) in patients with simple chest wall $\mathrm{TB}$ and $\overline{21.04} \pm 5.11$ (range: 7-80 days) in patients with composite chest wall TB.

The mean postoperative hospital stay was $\overline{21.91} \pm 7.89$ days (range: 7-62 days); $\overline{21.04} \pm 5.11$ days (range: 7-36 days) in patients with simple chest wall TB and $\overline{22.42} \pm$ 9.68 days (range: 8-62 days) in patients with composite chest wall TB. Significant differences were noted in preoperative anti-TB duration between patients of the two groups $(\mathrm{P}<0.0001)$. However, no significant differences were observed in postoperative hospital stay between the two groups $(\mathrm{P}=0.0868)$. Further details are presented in Table 2. A total of 234 patients (the remaining 29 were lost to follow-up) were followed-up for more than one year, of which 10 cases showed a recurrence (3.80\%). The incisions of the remaining patients healed well, and no symptoms, such as fever in the afternoon and fatigue, or redness and tenderness at the incision site, were observed.

Five patients had recurrence within one year. One such patient developed a chest wall mass outside the primary 
site during hospitalization, which was eventually confirmed as recurrence of chest wall TB. Pyogenic secretions were found in the incision of the original focus in one patient with diabetes mellitus and a poor physique, more than 3 months after the operation, which confirmed the recurrence of TB. Another patient developed a mass at the original incision site, 5 months after the operation, and recurrence of TB was confirmed by pathology. We later understood that this patient had discontinued the anti-TB drugs after discharge from the hospital.

One patient felt fatigue, night sweats, and low-grade fever in the afternoon, a month after the operation. Although the symptoms stabilized after intensive anti-TB treatment, recurrence of TB was considered. The last of five patients experienced pain at the original incision site and developed a slight protuberance from the site, which was eventually confirmed to be recurrence of the TB focus. The latter two patients had no active symptoms before the operation and took drugs regularly after the operation. However, we traced their medical histories and identified that the scope of the focus during the operation was larger than first expected. The cause of recurrence was considered insufficient to clear the TB lesions during the operation, and there may have been missed lesions. The other five patients had pain and discomfort at the original incision site more than one year after drug withdrawal and were considered to have recurrence for unknown reasons.

\section{Discussion}

Chest wall TB is characterized by a TB lesion that causes cheese-like necrosis, liquefaction, abscesses in the soft tissue of the chest wall, and destruction of the ribs, costal cartilage, and sternum. Most cases are secondary to pulmonary TB, which may occur in the lung, pleura, and/or vertebral body, but may also occur within the chest wall tissue. ${ }^{11,13}$ The treatment for chest wall TB includes simple TB drug chemotherapy, lesion puncture and drainage, and surgical treatment. ${ }^{14,15}$

It is usually difficult to completely eliminate most cases of chest wall TB by chemotherapy alone. Hence, surgical removal of the focus is essential. However, at present, there is no consensus regarding the optimal time of preoperative antituberculosis treatment and the best plan for perioperative treatment and management of chest wall TB. Understandably, some issues remain controversial.

At present, most of the data ${ }^{8,10,11}$ suggest that patients with chest wall TB require at least 2-3 months of preoperative anti-TB treatment to comprehensively control
TB infection and avoid postoperative TB recurrence. The purpose of preoperative anti-TB drugs is to control the number of TB bacteria within the focus and inhibit their growth, thereby minimizing the possibility of systemic dissemination of TB bacteria during the operation itself, and reducing the risk of low immunity during the perioperative period.

Some researchers ${ }^{16}$ believe that $\mathrm{TB}$ symptoms are obvious during the active stage of the disease, during which invasive surgical procedures can easily cause further aggravation of TB infection, and likely even lead to systemic spread. However, whether preoperative anti-TB treatment for more than 2-3 months can adequately control the number of TB bacteria within the focus, inhibit their growth, and increase surgical safety remains unknown. Furthermore, questions regarding whether an "early" operation affects the safety and prognosis of patients, and whether preoperative anti-TB treatment is indeed effective for all patients remain to be answered.

Relevant pharmacological studies ${ }^{10,17}$ have shown that the clinical efficacy of drug therapy on TB mainly depends on the drug concentration. Therefore, the concentration distribution of anti-TB drugs in the chest wall focus is critical for clinical efficacy. Most patients with chest wall TB form a sclerosing wall around the TB focus that affects the penetration of anti-TB drugs into the target site. This, in turn, leads to a failure to achieve optimal bacteriostatic and bactericidal concentrations. Furthermore, prolonged preoperative anti-TB treatment may also increase the potential rate of drug resistance. ${ }^{18}$ Therefore, some researchers have proposed the feasibility of a short term, enhanced, anti-TB regimen for chest wall TB and shortened duration of anti-TB medication before the operation. ${ }^{19}$

Patients with simple chest wall TB were given an ultrashort course of intensive anti-TB treatment before the operation. The data showed that while the postoperative recurrence rate and complications were not increased, the anti-TB treatment should be prolonged for patients with composite chest wall TB. In particular, patients with active TB showing symptoms, pleural effusion, and a positive sputum smear, as well as those with diabetes and a poor physique should have a prolonged duration of anti-TB treatment before the operation. Surgical treatment was performed after the focus of TB was stabilized and the physique improved. ${ }^{9}$

Overall, 28 patients received anti-TB medication for $>28$ days before surgery, all of whom were in the composite chest wall TB group. Among these patients, five with 
active TB received enhanced anti-TB medication for $>2$ months, and nine patients were either elderly or had complications related to diabetes and a poor physique. During the treatment, dynamic chest CT imaging showed that the active focus in the lung continued to be absorbed and was reduced, while the focus of chest wall TB persisted and showed no significant shrinkage. This was considered to be sclerosing walls formed around the chest wall TB focus, and the cause of a poor treatment response. The data for this group showed that the preoperative anti-TB duration for patients with composite chest wall TB was $\overline{21.04} \pm 5.11$ days, which was significantly higher than that for patients with simple chest wall TB $(\overline{12.82} \pm 5.87$ days $)$ $(\mathrm{P}<0.0001)$. Therefore, for patients with other types of TB, especially active pulmonary TB, prolonged duration of anti-TB treatment is still required to prevent postoperative recurrence, focal expansion, and TB dissemination.

The surgical principle of chest wall TB is "complete removal of the focus, elimination of the residual cavity, and pressure bandaging. ${ }^{, 6,7,11}$ If the TB infection was found to erode the ribs, costal cartilage, and sternum, or when the focus formed a dumbbell-shaped sinus, the damaged bones should be definitively removed, along with the ribs associated with the field of the lesions in order to minimize the occurrence of residual lesions. For patients with a large purulent cavity, the surrounding muscle tissue with good blood supply can be properly filled, while a smaller abscess can be eliminated by pressure bandaging. The presence of tissue with a poor blood supply and multiple foreign bodies, such as silk thread or a hemostatic sponge, within the purulent cavity should be avoided.

The main postoperative treatment for chest wall TB is the continuation and completion of the regular course of anti-TB medication. In the present study, 35 patients were hospitalized for $>28$ days after the operation. The likely causes are as follows: 28 patients were elderly ( $>60$ years); two patients with simple chest wall TB developed another TB lesion outside the original lesion, 20 days after the operation; and the remaining five patients had other TB-related infections before the operation. The postoperative hospital stay of patients with simple TB was $\overline{21.04} \pm 5.11$ days, while that of patients with other forms of TB was $\overline{22.42} \pm 9.68$ days $(\mathrm{P}=0.0868)$. Therefore, we believe that as long as the preoperative TB infection can be effectively controlled, the postoperative hospital stay will not be prolonged, regardless of the presence or absence of other TB-related complications.
The recurrence rate of $\mathrm{TB}$ was $3.80 \%$, and the main reason for the recurrence of chest wall $\mathrm{TB}$ was the lack of thorough clearance of the tuberculous focus during the operation. Some of the postoperative causes of recurrence include a large residual cavity with insufficient pressure bandaging, poor general condition of the patient, low immunity, and an irregular and/or incomplete course of anti-TB treatment. ${ }^{4}$ The recurrence rate within one year was lower than, or close to that reported in the literature. ${ }^{6,8}$ No significant differences were noted between the simple and composite chest wall TB groups. Therefore, preoperative, short term (2-3 weeks) anti-TB treatment is safe, reliable, and will not increase the postoperative recurrence rate. To control or reduce the recurrence of chest wall $\mathrm{TB}$, clinicians should pay close attention to completely removing the tuberculous focus during surgery, eliminating any residual cavity, and standardizing the complete course of anti-TB treatment, rather than simply prolonging the preoperative treatment time.

This was a retrospective study, with certain limitations. However, the concept of preoperative ultra-short-course chemotherapy, combined with surgery for the treatment of chest wall tuberculosis can still be proposed after the analysis of recent data, spanning 5 years at our hospital, and its safety has been verified. We will continue to improve relevant studies in the future.

\section{Ethics Statement}

This study was conducted in accordance with the principles of the Declaration of Helsinki, and the study protocol was approved by the Ethics Committee of the Henan Provincial Chest Hospital (No. 2019-05-012). Written informed consent was obtained from each participant.

\section{Funding}

The study was supported by a grant from the Henan Medical Science and Technology Research Program (No. 2018020567), as part of the Capital Characteristic Clinical Application Research project.

\section{Disclosure}

The authors report no conflicts of interest in this work.

\section{References}

1. García S, Combalía A, Serra A, et al. Unusual locations of osteoarticular tuberculosis. Arch Orthop Trauma Surg. 1997;116(6-7):321-323. doi:10.1007/BF00433981

2. Teo THP, Ho GH, Chaturverdi A, et al. Tuberculosis of the chest wall: unusual presentation as a breast lump. Singapore Med J. 2009;50(3): e97-99. 
3. Cataño J, Perez J. Tuberculosis abscess of the chest wall. Am J Trop Med Hyg. 2014;91(4):663. doi:10.4269/ajtmh.14-0063

4. Zhang N, Xu QZ, Fu XN, et al. Use of a pedicled omental flap in the treatment of chest wall tuberculosis. Ann Thorac Surg. 2012;93 (3):1010-1012. doi:10.1016/j.athoracsur.2011.08.053

5. Cho S, Lee E. Surgical resection of chest wall tuberculosis. Thorac Cardiovasc Surg. 2009;57(08):480-483. doi:10.1055/s-00291186066

6. Kim YT, Han KN, Kang CH, et al. Complete resection is mandatory for tubercular cold abscess of the chest wall. Ann Thorac Surg. 2008;85(1):273-277. doi:10.1016/j.athoracsur.2007.08.046

7. Cho KD, Cho DG, Jo MS, et al. Current surgical therapy for patients with tuberculous abscess of the chest wall. Ann Thorac Surg. 2006;81 (4):1220-1226. doi:10.1016/j.athoracsur.2005.10.044

8. Paik HC, Chung KY, Kang JH, et al. Surgical treatment of tuberculous cold abscess of the chest wall. Yonsei Med J. 2002;43 (3):309-314. doi:10.3349/ymj.2002.43.3.309

9. Wang G, Liu WX, Zhou XD, et al. Clinical analysis of effect of surgical treatment on 373 chest wall tuberculosis cases. J Clin Pulm Med. 2007;12(2):134-135.

10. Wang Z, Shi J, Geng G, et al. Ultra-short-course chemotherapy for spinal tuberculosis: five years of observation. Eur Spine J. 2013;22 (2):274-281. doi:10.1007/s00586-012-2536-0

11. Li L, Li Q, Xu SF, et al. Treatment Strategies of Tuberculosis. 1st ed. Beijing: People's Medical Publishing House; 2013:314-316.
12. WS 288-2017. Diagnosis for Pulmonary Tuberculosis. Beijing: National Health and Family Planning Commission of PRC; 2017.

13. Hsu HS, Wang LS, Wu YC, et al. Management of primary chest wall tuberculosis. Scand J Thorac Cardiovasc Surg. 1995;29(3):119-123. doi:10.3109/14017439509107217

14. Tsagouli P, Sotiropoulou E, Filippousis P, et al. Contribution of computed tomography guided percutaneous drainage of tuberculous cold abscesses adjunctive to pharmaceutical anti-tubercular treatment. Eur $J$ Radiol. 2012;81(3):562-565. doi:10.1016/j. ejrad.2011.02.048

15. Han YI, Zhao Q, Yu D, et al. Treatment of chest wall tuberculosis with transdermal ultrasound-mediated drug delivery. Exp Ther Med. 2015;9(4):1433-1437. doi:10.3892/etm.2015.2219

16. Guo D The Related Research of Different Days of Anti-Tuberculosis Before Surgery to Spinal Tuberculosis with Paraplegia Patients [dissertation]. Changsha: Central South University; 2010.

17. Zhen JR Different Days of Preoperative Chemotherapy on the Safety of Spinal Surgery and Prognosis for Spinal Tuberculosis [dissertation]. Wulumuqi: Xin Jiang Medical University; 2016.

18. Ge Z, Wang Z, Wei M. Measurement of the concentration of three anti-tuberculosis drugs in the focus of spinal tuberculosis. Eur Spine J. 2008;17(11):1482-1487. doi:10.1007/s00586-008-0778-7

19. Qian Y, Yang JC, Yuan YX. Clinical experiences of the surgical treatment of chest wall tuberculosis (120 cases report). Chin J Mod Operative Surg. 2011;15(5):364-365.

\section{Publish your work in this journal}

Infection and Drug Resistance is an international, peer-reviewed openaccess journal that focuses on the optimal treatment of infection (bacterial, fungal and viral) and the development and institution of preventive strategies to minimize the development and spread of resistance. The journal is specifically concerned with the epidemiology of antibiotic resistance and the mechanisms of resistance development and diffusion in both hospitals and the community. The manuscript management system is completely online and includes a very quick and fair peerreview system, which is all easy to use. Visit http://www.dovepress.com/ testimonials.php to read real quotes from published authors. 